\title{
La calidad en las instituciones de educación superior. Una mirada crítica desde el institucionalismo
}

\author{
Jacques-P., Victor; Boisier-O., Guy \\ La calidad en las instituciones de educación superior. Una mirada crítica desde el institucionalismo \\ Revista Educación, vol. 43, núm. 1, 2019 \\ Universidad de Costa Rica, Costa Rica \\ Disponible en: http://www.redalyc.org/articulo.oa?id=44057415029 \\ DOI: https://doi.org/10.15517/revedu.v43i1.30855
}

Esta obra está bajo una Licencia Creative Commons Atribución-NoComercial-SinDerivar 3.0 Internacional. 
La calidad en las instituciones de educación superior. Una mirada crítica desde el institucionalismo

\author{
Victor Jacques-P. \\ Universidad Austral de Chile, Chile \\ victorjacq@uach.cl \\ (DD http://orcid.org/https:// \\ orcid.org/0000-0002-7528-8374 \\ Guy Boisier-O. \\ Universidad Austral de Chile, Chile \\ guy.boisier@uach.cl \\ (iD http://orcid.org/https:// \\ orcid.org/0000-0002-1252-9273
}

The quality in higher education institutions. A critical view from institucionalism

DOI: https://doi.org/10.15517/revedu.v43i1.30855

Redalyc: http://www.redalyc.org/articulo.oa?id=44057415029

Recepción: 02 Noviembre 2017

Aprobación: 17 Diciembre 2018

\title{
Resumen:
}

En este ensayo se aborda el tema de la calidad en Instituciones de Educación Superior a la luz del enfoque institucionalista, teoría económica que surgió a mediados del siglo XX. A través de la presentación de un modelo sencillo y bajo supuestos que corresponden a una situación teórica, se derivan comportamientos óptimos que logran plantear buenos estándares en la calidad de la educación. Posteriormente, al considerar la presencia de algunas características que se observan en la realidad de estas instituciones tales como información asimétrica, oportunismo, problema de agencia, procrastinación, anomia y dificultad de medir el producto educativo, entre otras, se advierte sobre la amenaza, en ausencia de medidas correctivas, que exista una tendencia a que los funcionamientos de estos centros educativos deterioren su nivel de calidad.

Palabras Clave: Calidad, Educación superior, Institucionalismo.

\section{Abstract:}

This essay explores approaches the topic of quality in Higher Education Institutions under the framework of an institutional approach, an economic theory that emerged in the mid-twentieth century. Through the presentation of a simple model and under assumptions that correspond to a theoretical situation, optimum behaviours are derived and these behaviours present good standards in the quality of education. Later, when considering the presence of some characteristics that are observed in the reality of these institutions such as asymmetric information, opportunism, agency problem, procrastination, anomie and difficulty in measuring the educational product, among others; it warns about the thread, in absence of corrective measures, that there exists a tendency for the operations of these educational centers to deteriorate their level of quality.

KEYWORDS: Quality, Higher education, Institutionalism.

\section{INTRODUCCIÓN}

En Chile, en el año 2011 hubo un hecho sobresaliente, el estudiantado universitario se volcó a las calles para manifestar abiertamente la necesidad de un cambio en la educación. Específicamente demandó con mucha fuerza y entusiasmo, una reforma en la educación superior que instalara en el país una educación pública, gratuita y de calidad. La administración de la presidenta Bachelet incluyó esta petición en su programa

\section{NotAS DE AUTOR}


de gobierno. Para ello, la reforma tributaria impulsada simultáneamente por las autoridades serviría de complemento a la reforma educacional. Se suponía que la nueva tributación iba a proporcionar suficientes recursos para garantizar una educación universitaria gratuita. A la fecha, esta demanda se ha logrado con un éxito relativo. Sin embargo, el segundo aspecto, la calidad de la educación, actualmente constituye una gran interrogante.

Recogiendo este hecho contingente, este trabajo aborda la calidad de las Instituciones de Educación Superior (IES) poniendo como eje de análisis el rol que cumple la institucionalidad que prima en estas organizaciones. Se entenderá la calidad como la capacidad que tienen las IES de cumplir verdaderamente con sus propósitos a través de acciones y actividades acordes a dichas intenciones y de verificar este cumplimiento ajustando las acciones en función de los resultados obtenidos de tal verificación (Comisión Nacional de Acreditación [CNA], 2016).

El artículo se inspira en los conceptos elaborados por la corriente institucionalista (Coase, 1996; North, 2014; Williamson, 1989). Siguiendo a North (2014), las instituciones son constructos ideados por los individuos para dar forma a la interacción humana. Comprenden las reglas, las costumbres, los incentivos, los desincentivos y los mecanismos de cumplimiento para hacer cumplir los acuerdos. En definitiva, se componen de una parte formal (constituciones y leyes) e informales (códigos de conducta).

El propósito es esclarecer cómo el marco institucional puede afectar el logro de un buen proceso de enseñanza-aprendizaje al interior de una institución académica, cuestión determinante en la calidad de la formación de la población estudiantil.

En el ámbito macroeconómico, un aporte fundamental de la corriente institucionalista fue sin duda destacar la importancia que adquieren las instituciones en el desarrollo económico de un país. Desde esta perspectiva, no basta que una nación posea ciertos factores productivos, ya sea capital físico o humano, tecnología o recursos naturales para garantizar una senda de crecimiento. Se requiere un elemento adicional, poseer buenas instituciones, esto es, adecuadas reglas del juego junto a un aparato del Estado encargado de velar para que estas se cumplan y especialmente, en la sociedad contar con cultura, costumbres y valores alineados con la normatividad vigente. En definitiva, se sostiene que las instituciones importan y en muchos casos ello explicaría la diferencia en el crecimiento de algunos países y el estancamiento de otros.

A nivel microeconómico, Coase (1996), al preguntarse por qué existen las firmas o empresas como unidad económica, destacó que estas surgen para evitar los costos de transacción que resultan en las relaciones mercantiles, en un mundo donde existe asimetría de información, incertidumbre y comportamiento oportunista de los agentes. Bajo estas circunstancias, usar el mercado involucra un costo. Por tanto, se desprende que resulta más eficiente recurrir a la coordinación administrativa, jerárquica al interior de la empresa, como un medio eficaz para asignar los recursos, en vez de utilizar la coordinación descentralizada de mercado. En cuanto a los límites de crecimiento de la firma, estos están dados finalmente por los problemas de coordinación, burocracia y de administración que aparecen cuando la empresa aumenta de tamaño. En ese momento entonces, resulta beneficioso pasar de la coordinación administrativa a la coordinación de mercado.

En ambos casos, tanto a nivel macro como micro, se evidencia que una adecuada institucionalidad es una de las condiciones necesarias para que los procesos económicos se desarrollen de mejor manera ${ }^{[1]}$. Siguiendo esta misma perspectiva de análisis, en este trabajo se discute acerca del tipo de institucionalidad el cual es conveniente que exista al interior de una institución académica, para que ayude a garantizar que el proceso de enseñanza-aprendizaje se realice dentro de un marco apropiado. El motivo de este propósito es alertar frente a la amenaza de que se instale en un futuro un marco normativo laxo e inadecuado y además una cultura con costumbres y hábitos tanto a nivel de estudiantes, docentes y directivos que obstaculizan un buen proceso formativo $^{[2]}$. 


\section{EL PROCESO FORMATIVO Y SU INSTITUCIONALIDAD}

La consecución de un grado o título universitario requiere que la población estudiantil acredite los conocimientos y competencias que la profesión exige. Ello obliga a que el alumnado se someta a un proceso de aprendizaje que requiere de años de dedicación. ¿Cuáles son los caminos, las vías institucionales y organizacionales que pueden existir para adquirir estos conocimientos? A continuación, se proponen dos opciones extremas.

\section{UN ESQUEMA DESCENTRALIZADO}

Siguiendo la línea argumental de Coase en su artículo acerca de por qué existen las empresas ${ }^{[3]}$, una alternativa es que este proceso de instrucción se realice de manera absolutamente descentralizada, es decir, a través del mercado. Esta alternativa requiere que cada estudiante se informe de los contenidos que es necesario aprobar y adquiera en el mercado los materiales (libros, videos, artículos, entre otros) los cuales requiere para aprender y contrate además a algún especialista en la materia, por un número determinado de horas, para despejar dudas e interrogantes que surjan de su aprendizaje. Claramente este método de adquirir conocimientos le resultaría difícil y engorroso, pues se encuentra en desventaja para enfrentar el mercado de enseñanza al no disponer de información plena, además su capacidad de decisión es limitada (Simon, 1962) [4]. Ciertamente carece de los conocimientos y de la experiencia para abordar esta tarea de manera racional y autónoma. En términos breves, el mercado del conocimiento no se puede afrontar sin conocimiento. El tipo de bien y servicio específico que se transa en este mercado reúne las características de un mercado de información asimétrica, incertidumbre y susceptible de tener costes de transacción ${ }^{[5]}$.

\section{UN ESQUEMA DE JERARQUÍA}

Una manera más eficiente de realizar el proceso de aprendizaje es que existan Instituciones de Educación Superior que impartan distintas profesiones y que la población estudiantil se matricule en ellos eligiendo su carrera de preferencia. Por un lado, las unidades de educación superior garantizan que cada profesión/ especialidad está estructurada con base en una malla curricular o programa de estudios que certifica la obtención de un determinado perfil de egreso con los conocimientos y competencias preestablecidas. Por su parte, el estudiantado inscrito es agrupado en cursos y parte de su proceso formativo lo desarrollan en aulas, conducidas y dirigidas por personal docente que se circunscribe a contenidos, programación y horarios predeterminados. Este método para adquirir conocimientos y competencias, sin duda hace que el proceso sea más eficiente, expedito y fácil para el alumnado ${ }^{[6]}$. Se elimina la incertidumbre de qué es necesario aprender y en qué orden. Es decir, no existen en este aspecto mayores costos que significan recurrir al mercado para llevar a cabo el aprendizaje ${ }^{[7]}$.

Sin embargo, existe un problema latente. Bajo este esquema de jerarquía o de administración de la enseñanza al interior de una IES, no existen las garantías inherentes para que este proceso se realice dentro de los estándares de calidad requeridos por los sistemas locales de acreditación de las instituciones. El desafío consiste entonces en que las instituciones logren ser eficientes, transformen las capacidades del estudiante, le otorguen al alumno valor agregado durante el proceso de aprendizaje y aseguren por consiguiente la calidad de la enseñanza superior. 


\section{FACTORES INSTITUCIONALES QUE CONTRIBUYEN A UN BUEN DESEMPEÑO DE UNA INSTITUCIÓN DE EDUCACIÓN SUPERIOR}

El objetivo de este apartado es proponer un esquema basado en algunos elementos determinantes en el aseguramiento de la calidad de una IES. Estos pueden deducirse de los criterios de acreditación institucional de la Comisión Nacional de Acreditación de Chile (CNA, 2016) los cuales se orientan a establecer una situación ideal en los diferentes niveles de funcionamiento de una IES, cuestión que se proyecta en cada uno los elementos y ecuaciones que proponemos a continuación.

\section{Elementos de una institucionalidad que apoyan una buena gestión y control de la organización}

Para que una IES cumpla con las condiciones que aseguren un adecuado funcionamiento del sistema y garantice una adecuada calidad, el centro de enseñanza debe cumplir con algunas condiciones deseables. A continuación, se bosquejan algunos elementos institucionales básicos:

a) Marco institucional. En primer término, para el logro de su propósito institucional, las IES deben contar con un conjunto de estatutos, reglamentos, normas y procedimientos establecidos, además de una estructura organizacional ad-hoc, que en términos generales definirán el marco institucional. En dicho ordenamiento debe precisarse claramente lo que se puede hacer y lo que no es permitido realizar en el transcurso de este proceso educativo. Esta estructura reglamentaria debe ser conocida y plenamente aceptada e internalizada por todos los miembros de la comunidad académica.

$$
C=f(M I)
$$

$+$

La ecuación (1) ${ }^{[8]}$ señala que la Calidad (C) de las IES depende directamente del adecuado diseño y reglamentación del Marco Institucional (MI) ${ }^{[9]}$.

b) Programación de actividades. Con base en este marco general, la planificación y programación de las actividades del proceso de enseñanza-aprendizaje quedan públicamente establecidas y adquieren un carácter definitivo. Dicha normativa debe ser conocida por directivos, docentes, estudiantes y personal de apoyo. Entre otros aspectos, cabe destacar los perfiles de egreso, los contenidos, calendarización de actividades, niveles de exigencia y requisitos de aprobación. Esto significa que existe información simétrica por todos los agentes involucrados en el proceso.

$$
C=f(P, I S)
$$

$$
+,+
$$

La ecuación (2) expresa la calidad del centro en función directa de la programación de actividades (P) y la existencia de información simétrica (IS) entre los agentes de las normas establecidas ${ }^{[10]}$.

c) Cultura imperante en el centro educativo. Los valores (V) que prevalecen en la institución académica son funcionales al sistema y facilitan el logro de los objetivos propuestos institucionalmente ${ }^{[11]}$. Existe una alta valoración por desarrollar las actividades de la mejor manera posible. Aquellos individuos que destacan 
(docentes, directivos, estudiantes y personal de apoyo) son reconocidos por la comunidad que compone las IES. Los docentes en lo fundamental tienen como prioridad desarrollar clases con apego a los contenidos establecidos y fomentando la motivación en los estudiantes ${ }^{[12]}$ por su parte la población estudiantil valora una buena formación y están atentos a que la institución garantice el cumplimiento del perfil de egreso comprometido; el clima organizacional es proclive para generar incentivos para el trabajo académico. En definitiva, existe coherencia entre valores, propósitos y desempeño al interior de la organización.

$$
C=f(V)
$$

$+$

La ecuación (3) resalta que la calidad de las IES depende positivamente del grado de alineamiento y pertinencia de los valores que ostentan los integrantes de él.

\section{FUnCIONAMIENTO Y RESULTADOS ESPERADOS DEL SISTEMA}

En caso de cumplirse este esquema óptimo, es posible prever los siguientes atributos del funcionamiento de las IES:

- La información fluye en las interacciones académicas. El profesorado facilita todos los medios docentes y la información necesaria.

- Se incentiva un clima colaborativo al compartir conocimientos y materiales de trabajo.

- Los plazos de entrega de compromisos tanto de docentes como de estudiantes son cumplidos.

- El estudiantado conoce la calendarización de sus obligaciones académicas, así como las fechas de compromisos y trabajos que les induce a programar sus acciones.

- El alumnado está motivado por aprender, considera que los conocimientos y las distintas instancias del trabajo académico que desarrolla en los programas que cursa son útiles para su formación como persona y para un adecuado desenvolvimiento posterior en el mercado laboral. Cualquier desviación o incumplimiento de los programas establecidos es expuesto por el estudiantado y corregido inmediatamente por la autoridad académica.

- En ese contexto, el uso y la asignación adecuada del tiempo en actividades orientadas a su formación integral es un expediente fundamental.

Definido este escenario deseable, a continuación, se mencionan los resultados posibles de esperar ${ }^{[13]}$. Las actividades académicas se desarrollan en forma expedita. El sistema en su conjunto funciona de manera ordenada y con total certidumbre, operando los mecanismos de autorregulación. No existen las condiciones del entorno para que los actores se desvíen de su función principal. Por ejemplo: a) el ausentismo docente a las clases es mínimo y los programas de estudios son realizados a cabalidad; b) no es necesario invertir tiempo en conseguir materiales de estudio o trabajo pues existe total disponibilidad de estos; c) cada estudiante solo destina su energía y tiempo a aquella función que le corresponde realizar. Es así que en este esquema no tiene cabida desalinear acciones, desarrollar comportamiento oportunista que evite el trabajo o busque conseguir prerrogativas personales. En este marco, cualquier actividad fuera de la norma resulta inconducente. 


\section{FACTORES QUE ATENTAN PARA UN BUEN FUNCIONAMIENTO DEL SISTEMA EDUCATIVO}

Como se señaló anteriormente, en el mundo real, en la cotidianidad de las IES, se aprecian ciertos elementos que llevan a considerar que existe una cierta brecha de calidad, del modelo esbozado. Al respecto, es preciso destacar lo que menciona Fernández-Lamarra (2012) sobre la diversificación institucional de IES en América Latina:

con universidades de alto nivel académico, equivalentes a las mejores del mundo -como la UNAM de México, la USP de Brasil y la UBA de Argentina- y muchas otras -la mayoría privadas- con bajísimos niveles de calidad, quizás la peores a nivel mundial. Son las denominadas "universidades garaje" en Colombia y otros países andinos o "universidades patito" en México, por ejemplo (p. 665).

En caso de no disminuir esta brecha, ello sin duda resentiría la eficiencia del sistema y, por ende, constituye un problema al fortalecimiento de la calidad en la educación superior en América Latina.

\section{Factores específicos que AfEctan eL FUnCionamiento de LAS IES}

Por lo general, las IES que han sido acreditadas por los sistemas locales de acreditación, cuentan con una adecuada definición de objetivos y propósitos y un conjunto de normas y reglamentos que ordenan la organización para alcanzar dichos fines. Sin embargo, su cumplimiento requiere que todos los miembros de la comunidad académica internalicen plenamente sus objetivos y se identifiquen a cabalidad con los medios necesarios para lograrlo, cuestión elemental de todo sistema de gestión y aseguramiento de la calidad. A continuación, se señalan algunos factores que pueden afectar la excelencia de una IES:

\section{Establecimiento de estándar por las autoridades nacionales de Educación}

En el caso de Chile, la institucionalidad de educación superior utiliza estándares de eficiencia y calidad para la asignación de recursos públicos y, eventualmente, la acreditación de las universidades en sus distintos niveles. Dentro del conjunto de elementos que se consideran para la evaluación, interesa destacar dos elementos. Las universidades son evaluadas favorablemente en la medida que presentan buenos ratios relacionados con a) bajas tasas de deserción y b) titulación oportuna ${ }^{[14]}$. Estos requerimientos para ser cumplidos exitosamente constituyen condicionantes que en algún grado pueden llevar a disminuir los niveles de exigencia académica.

$$
C=f(I D T)
$$

La ecuación (4) expresa la calidad en función inversa de la exigencia asociada a la meta de cumplir con indicadores de deserción y titulación (IDT).

\section{El gobierno de las IES}

El sistema de gobierno que caracteriza a las IES, a pesar de presentarse muchas veces como piramidal jerarquizado, está constituido por elementos que contribuyen a un funcionamiento más horizontal por las relaciones de proximidad relacional-social y cognitiva ${ }^{[15]}$ (Bouba-Olga y Grossetti, 2008; Mora y Moreno, 2010) entre directivos y académicos. Tal hecho es posible de relevarlo en aquellas instituciones donde existe 
un sistema de elección de pares para el ejercicio de cargos directivos (Rectoría, Decanatura, Dirección, entre otros), siendo académicos que transitoriamente ocupan esa función; ello significa que ejercen como directivos de sus colegas solamente por un lapso determinado. Con posterioridad, al cumplir el periodo asignado al cargo, se reintegran a sus funciones docentes junto al personal académico. Esta función temporal como directivo constituye un elemento que impide que se aplique en plenitud la relación de dependencia al interior de la organización pues se privilegia también la armonía presente y futura, las relaciones laborales con los pares y también en algunas IES los vínculos de amistad. Este factor naturalmente puede contribuir a que exista una relajación en el grado de rigurosidad en la aplicación de las normas y reglamentos.

$$
C=f(E C)
$$

La ecuación (5) enuncia la calidad como una relación inversa a la jerarquía organizacional influenciada por el efecto colega ${ }^{[16]}$.

\section{El problema de agencia al interior de las IES}

Se asume que las autoridades académicas responsables de la institución son las que fundamentalmente están interesadas en que la actividad de enseñanza-aprendizaje se lleve a cabo de manera óptima. Para su ejecución, se realizan contratos con docentes quienes se encargan de conducir la actividad académica. Ciertamente, el proceso de aprendizaje depende en gran medida en el desempeño que exhiba por un lado el personal docente y por otro el estudiantado. Es claro, si cada agente realiza su función de manera muy satisfactoria, alineados en función de los propósitos de la institución, la probabilidad que el resultado de la actividad sea exitoso es muy alta. Sin embargo, existen elementos que pueden lesionar o desviar este proceso.

El personal directivo de las IES poseen información asimétrica, lugar donde se instalan las bases del aprendizaje. A ellos les resulta dificultoso obtener toda la información necesaria, así, por ejemplo, no conocen a cabalidad: a) el programa verdaderamente impartido en cada asignatura; b) la calidad del sistema de evaluación aplicados por el docente a los estudiantes; c) las técnicas pedagógicas aplicadas al interior del aula; d) el grado de profundidad de los conocimientos impartidos; e) el nivel de comprensión de las materias alcanzado por los estudiantes, entre otras. En definitiva, las salas de clase constituyen, en términos de Coase, una caja negra para los directivos. Habitualmente, la información que obtienen a través de indicadores estandarizados se limita a cuestiones de forma, tales como conocer el número de estudiantes que pasan de una promoción a otra y cuáles son sus calificaciones, pero no disponen del conocimiento pleno de la eficacia esencial del proceso de aprendizaje ${ }^{[17]}$. En otros términos, bajo información asimétrica o ausencia de simetría de información, brotan posibles comportamientos oportunistas (Williamson, 1989) de los docentes y estudiantes, y en ausencia de efectivo control interno (CI), no es posible garantizar que los indicadores académicos constituyan un fiel reflejo del verdadero resultado de la actividad de enseñanza. El comportamiento oportunista se refiere a aquellos comportamientos donde se privilegia el interés individual por sobre el de la organización y donde los individuos transmiten información incompleta o distorsionada con el fin de distorsionar, confundir u ocultar aspectos de la misma.

El inconveniente planteado, puede ser analizado del punto de vista del problema de agencia al interior del centro de enseñanza (Jacques y Saona, 2011). 


$$
C=f(O, C I)
$$

Dada una cierta asimetría de información, la calidad de las IES se deteriora a medida que existe mayor oportunismo y una ausencia de control interno (ecuación 6).

\section{La especificidad de las actividades educativas ayuda a que surjan relaciones desalineadas en el proceso de enseñanza-aprendizaje}

Una particular característica del sector educación, es que el producto (i.e. conocimientos, competencias, habilidades) que se genera al interior de las IES, es difícil de medir. Esto se debe a que el resultado no constituye un elemento totalmente objetivable ${ }^{[18]}$. Este hecho genera dos imprecisiones que, sin duda, dificultan la actividad de evaluación y control del proceso de enseñanza-aprendizaje, tanto por parte de los directivos como a nivel del aula por parte de los docentes.

1. A nivel de directivos. ¿Cómo los directivos pueden discriminar en qué semestre una misma asignatura obtuvo un mayor o menor logro? ¿Cómo pueden comparar de manera inequívoca en cuál de dos materias impartidas paralelamente se alcanzó un mejor resultado en el aprendizaje? Si bien existen instrumentos de medición factibles de control de los procesos académicos, ellos no corrigen en su totalidad el problema de la asimetría de información. En efecto, esta última junto con la racionalidad limitada que enfrentan los directivos (Simon, 1962), no es solo una particularidad de ellos, sino que, asimismo, al interior de una asignatura, el problema de medición del aprendizaje también se presenta para el académico que imparte la asignatura.

2. A nivel de aula. Para llevar a cabo el proceso de evaluación, el docente no cuenta con una métrica invariable exenta de error que permita medir con precisión los conocimientos y competencias adquiridas por el estudiante durante y al final del curso. En este ámbito, inexorablemente existe un grado de subjetividad y de aproximación al objeto medido.

Esta falta de claridad inherente en la medición del producto constituye un factor que, en alguna medida, da pie para que los agentes que participan en el proceso de enseñanza-aprendizaje desarrollen un comportamiento oportunista, es decir, se desalineen con respecto a los propósitos de la organización. En efecto, con asimetría de información, existe un margen para que docentes y alumnos realicen estrategias de carácter oportunista, dirigidas a disminuir el nivel de actividad de una asignatura a fin de privilegiar otras acciones, por ejemplo, de interés personal o particular.

\section{Factores de Carácter general. La posible tendencia haCia Un Grado de ANOMia EN LAS IES}

Para analizar si existe una cierta tendencia a no respetar cabalmente la institucionalidad en las IES, se considera el concepto de anomia desarrollado por Merton (1995). Este autor, en su estudio, destacó en las estructuras sociales dos elementos fundamentales. a) El primero incluye "los objetivos, propósitos e intereses culturalmente definidos, sustentados como objetivos legítimos por todos los individuos de la sociedad, o por individuos situados en ella en una posición diferente" (p. 210) y b) El segundo elemento, se refiere a 
"los modos admisibles de esos objetivos" (p. 210) que son definidos, regulados y controlados por la misma estructura cultural. Para el autor, la anomia se produce entonces, cuando existe una discordancia entre medios y fines. Se trata de aquellas situaciones en que los individuos, con la intención de lograr sus objetivos particulares, despliegan diversas acciones que no concuerdan con el marco institucional vigente. Esto es, no respetan o no someten íntegramente sus medios al orden establecido.

$$
C=f(A)
$$

La ecuación (7) destaca el hecho que a medida que exista un mayor grado de anomia en una IES, menor calidad presentará esta.

En este mismo sentido, en una IES los agentes participantes del proceso educativo deben afrontar un conjunto de objetivos y restricciones, estas últimas impuestas por el sistema interno y externo. Bajo esas circunstancias, para los participantes respetar completamente las normas vigentes establecidas socio culturalmente, genera una cierta tensión cuando buscan lograr otros propósitos individuales ${ }^{[19]}$. Ello lleva a buscar caminos alternativos, atajos, que atentan contra la calidad del proceso de enseñanza -aprendizaje. Algunos ejemplos ilustran lo anteriormente señalado:

a) Como ya se ha señalado, la necesidad de alcanzar ciertos indicadores establecidos externamente a las IES, como son los exigidos por los sistemas de acreditación, es un factor que tiende a influir para que directivos y académicos, con el propósito de conseguir el mejoramiento de las exigencias diferentes a las formativas (por ejemplo, alcanzar adecuados indicadores en la tasa de abandono y duración media de los estudios), tiendan a acomodar las acciones, sacrificando aspectos que influyen en la excelencia y la rigurosidad del proceso formativo.

b) Existen incentivos subyacentes para los docentes de bajar las exigencias académicas. Con información asimétrica, en ausencia de un cierto control tanto por parte de los directivos como de los estudiantes, los docentes pueden verse inclinados a desarrollar un trabajo sin grandes complicaciones. Un sistema de enseñanza y de evaluaciones carente de exigencias elevadas, permite garantizar altos índices de aprobación y evita en el día a día descontentos y una mayor preocupación y trabajo con los estudiantes.

c) Una observación de la realidad en las universidades hoy permite sostener que las nuevas generaciones disponen de una mayor cantidad de distracciones, lo que los induce a tener una actitud más satisfaciente que optimizadora ${ }^{[20]}$ en sus actividades académicas. También se aprecia una relación entre directivos, docentes y alumnos más horizontal que en décadas anteriores. En algún grado estos factores posibilitan el desarrollo de mecanismos por parte de los estudiantes orientados a conseguir mejorar las calificaciones, sin recurrir a mayor intensidad de estudios. Estos caminos distintos al estudio se asocian con: tramites de conseguir información privilegiada acerca de las características de las exigencias, presentación de justificativos, solicitud de postergación de compromisos académicos, modificación de fecha de evaluaciones, de regateo con los docentes acerca del nivel de las calificaciones, de la cantidad de evaluaciones, entre otras. En definitiva, hay un grado de desviación de actividades hacia la búsqueda de transparentar información, acciones de lobby y desarrollo de influencia con directivos y docentes que ayudan a salvar las exigencias académicas mediante formas alternativas a la dedicación al estudio. El problema que demanda atención es que en la medida que se incrementen estas prácticas, el proceso de enseñanza-aprendizaje pierde eficiencia y calidad. 


\section{FACTORES DE DESMOTIVACIÓN QUE CONTRIBUYEN AL DESALINEAMIENTO EN EL PROCESO DE ENSEÑANZA-APRENDIZAJE}

Previo a considerar la tendencia a desalinearse de los agentes que participan en el proceso enseñanzaaprendizaje, es necesario resaltar la importancia del marco general en que estos se desenvuelven. En este sentido, es necesario destacar el clima organizacional imperante en las IES, el cual está vinculado con su tradición y al conjunto de reglas que han prevalecido. En aquellas instituciones con mayor solidez y resguardo, existirá una tendencia a la excelencia. En la medida que las normas y cultura resulten más permisivas, habrá motivo para desarrollar, a través del tiempo, conductas que se alejen de la exigencia y, a la vez, se desarrollarán procedimientos alternativos para lograr objetivos individuales. En definitiva, en un contexto propicio, tanto docentes como estudiantes pueden tener incentivos para reducir el nivel de exigencia del proceso enseñanzaaprendizaje y con ello afectar su calidad.

\section{Docentes}

La pérdida de aliciente del profesorado con respecto a la enseñanza, se puede asociar a al menos a los siguientes elementos:

a) Por lo general, en las universidades se concibe la docencia como la obligación básica que debe cumplir un académico como retribución a su remuneración. Los incentivos adicionales se orientan entonces preferentemente en la investigación, publicación y asistencias a congresos. De este modo, cada docente en su jornada laboral enfrenta una disyuntiva entre docencia e investigación, lo cual lleva en ocasiones a privilegiar esta última (Fernández-Lamarra y Coppola, 2008).

b) En la actualidad, el cuerpo docente percibe que el estudiantado exhibe un menor grado de interés por aprender los contenidos que comprende la malla curricular o programa de estudios. El menor interés de los estudiantes, contribuye a su vez, a que los académicos se desmotiven al no sentir la suficiente retroalimentación ${ }^{[21]}$.

\section{Estudiantes}

Diversos factores pueden influir para que los estudiantes presenten algún grado de desmotivación al estudio, afectándose con ello la calidad del proceso educativo. A continuación, se señalan algunas de estas causas.

a) En la medida que los estudios superiores en el mundo moderno comienzan a ser considerados por los estudiantes principalmente como un medio necesario para acceder a rentas altas en el mercado laboral, esta idea necesariamente tiende a conspirar con el interés y el deseo por parte de los estudiantes de adquirir conocimientos para ampliar su visión de la sociedad y de la cultura.

b) Un porcentaje de los estudiantes confía que, si bien el rendimiento medido por las notas en los estudios superiores importa, no es el único elemento que incide a la hora de obtener una fuente laboral, factores como los contactos personales, habilidades relacionales, capital social, entre otras variables, también influyen (López, 2016). Lo anterior, puede llevar a estimar por parte de los estudiantes que no es imprescindible obtener buenas calificaciones en el programa de estudio para lograr una buena inserción laboral. En otros términos, en los años de estudiante, bastaría realizar sólo el esfuerzo necesario que se requiere para lograr un nivel satisfactorio.

c) Estudiar es una actividad que significa sacrificar otras actividades que pueden ser placenteras i.e. el ocio, compartir con familiares, amigos, novia (o), hacer deporte entre otras, de este modo, desde un punto de vista racional, la decisión de estudiar resulta conveniente cuando la compensación o el 
premio justifica el costo de oportunidad. No obstante, los individuos muchas veces actúan con una racionalidad limitada (Simon, 1962; Kahneman y Tversky, 1973). De este modo, algunos estudiantes pueden optar por satisfacciones presentes, en desmedro de su formación futura, lo cual los hará subjetivamente sobreestimar o subestimar el rendimiento de un estudio de última hora y también las probabilidades de éxito en el certamen futuro. Este raciocinio, sea consciente o no, lleva que en definitiva tiendan a esforzarse menos en su trabajo como estudiante.

d) La carencia de un método sistemático de estudio a través del tiempo por parte de los estudiantes se puede abordar considerando la existencia de procrastinación en ellos ${ }^{[22]}$. Esta noción analiza porqué las personas son incapaces de autorregularse en la realización de sus obligaciones, existiendo en ellas la tendencia a postergar sus tareas ${ }^{[23]}$ A la hora de enfrentar una tarea, existen diversos factores que inhiben su realización; estos tienen su origen en procedencias de índole emocional y conductuales, tales como la desmotivación, los niveles de auto exigencia, depresión, temor al fracaso, incapacidad de tomar decisiones, entre otras. También influye la falta de un objetivo o estimulo suficiente que justifique una conducta autorregulada (Quant y Sánchez, 2012).

$$
C=f(D)
$$

La ecuación (8) expresa como la calidad de una IES se deteriora, conforme exista un mayor nivel de desmotivación por parte de docentes y estudiantes en el proceso de enseñanza-aprendizaje.

En síntesis, la calidad de las IES está, al menos, en función de las siguientes variables (ecuación 9):

$$
\begin{gathered}
C=f(M I, P, I S, V, I D T, E C, O, C I, A, D) \\
+,+,+,+,-,-,-,+,-,-,
\end{gathered}
$$

\section{MODELO DE ANÁLISIS}

Las variables que contribuyen o afectan la calidad de una IES pueden consolidarse en un modelo simple de análisis que se expresa en los Gráficos 1 y 2 . En el eje vertical o eje de ordenadas se ubica la variable calidad (C) de las IES, la cual está en función de la variable independiente que se ubica en el eje horizontal o eje de abscisas que representa los valores de una IES, denominada (V). Esta última variable representa el conjunto de valores que condicionan a los participantes a proporcionar un determinado esfuerzo en el proceso de enseñanzaaprendizaje. En la medida que (V) aumenta, se produce una mejora cualitativa de estos valores y por ende una mayor calidad en el proceso educativo. De este modo, la gráfica $\mathrm{CV}$, que asocia para cada ascenso de (V) el correspondiente nivel de (C) es de pendiente positiva. Como parámetros de esta curva, aparecen las otras variables que también moldean e influyen en las actuaciones y desempeños de los distintos agentes que participan en proceso de enseñanza- aprendizaje. El marco institucional, dado que en el corto plazo es un dato, se representa por una gráfica vertical, allí se encuentra el conjunto de parámetros que definen el contexto y reglas del juego que imperan en una IES.

El Gráfico 1, muestra, en primer lugar, que el nivel de calidad se determina como resultado de la existencia del conjunto de valores que adoptan las variables consideradas en una IES. En segundo lugar, dado un marco 
institucional y un cierto nivel de valores de las variables expuestas, se aprecia cómo se afecta la calidad de una IES, cuando aumenta el "efecto colega" o cuando aumentan los niveles de oportunismo de docentes y/o estudiantes o bien, cuando aumenta la anomia o la desmotivación en los agentes. El resultado en todos estos casos, es que las IES presenta un menor nivel de calidad de la educación ${ }^{[24]}$.

\section{Gráfico 1: Determinación del nivel de calidad en una IES y efecto del cambio del efecto colega.}

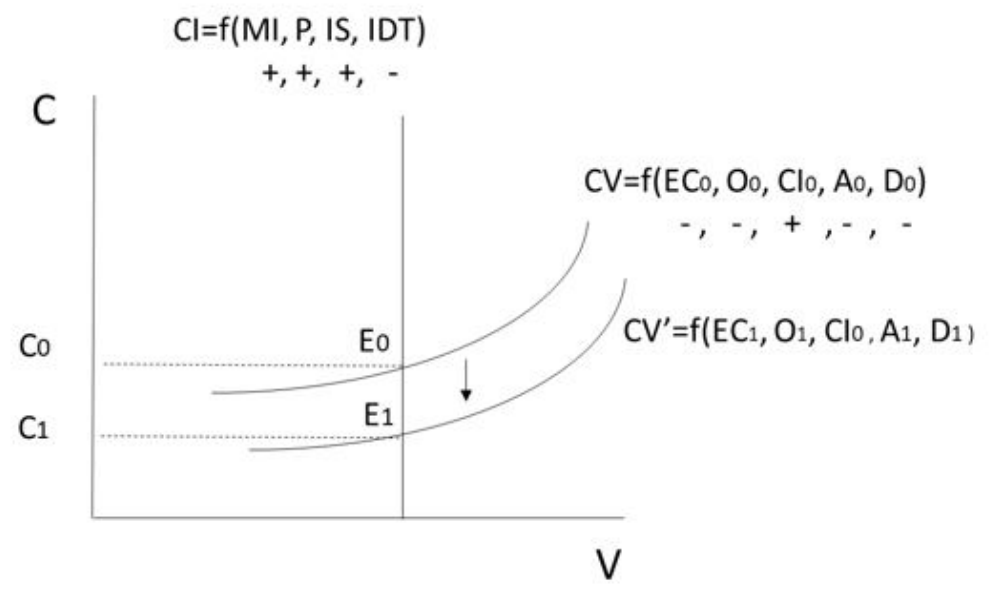

Fuente: Elaboración propia

\section{Gráfico 1: Determinación del nivel de calidad en una IES y efecto del cambio del efecto colega}

Fuente: Elaboración propia

En el Grafico 2, se presenta la situación que acontece cuando las IES modifican favorablemente su marco institucional, que por cierto incluye los valores de la institución. Esta modificación significa claramente una mejora en la calidad educativa. 


\section{Gráfico 2: Efecto de un perfeccionamiento de las instituciones en el nivel de calidad de una IES}

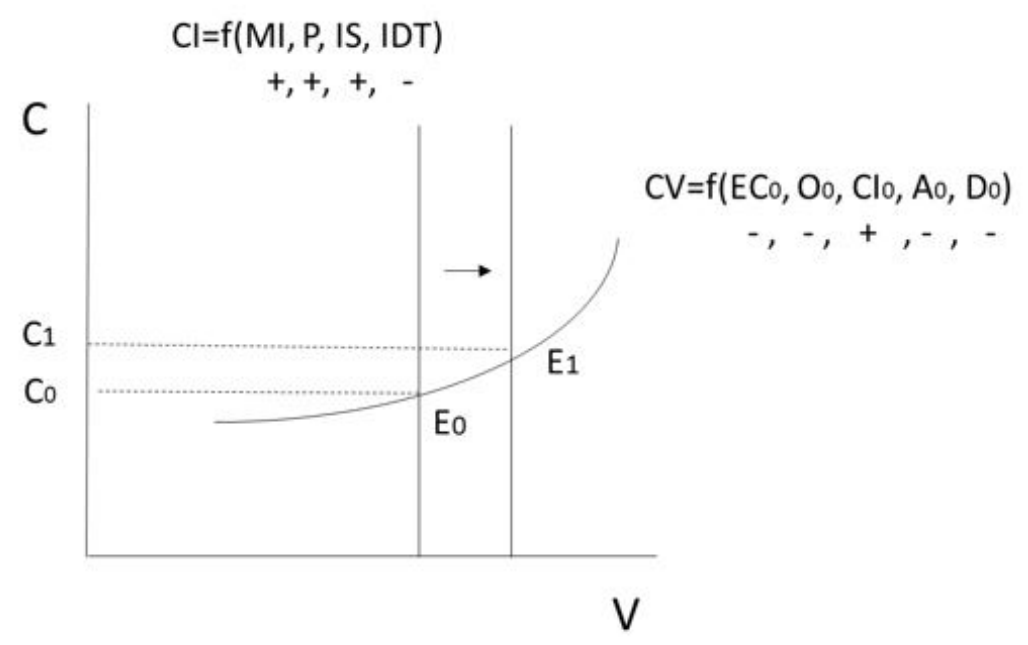

Fuente: Elaboración propia

Gráfico 2: Efecto de un perfeccionamiento de las instituciones en el nivel de calidad de una IES Fuente: Elaboración propia

\section{Conclusiones}

En este artículo se sostiene que la institucionalidad que exista en una IES cumple un rol fundamental, sobre todo en lo que respecta al aseguramiento de la calidad de la organización. Sin embargo, debe tenerse en cuenta que el proceso educativo presenta características muy específicas, que por su naturaleza no son fácilmente observables. De allí que el tipo de gestión institucional que se aplique en las IES es determinante. En caso que la gestión no se realice de manera propicia, ello puede contribuir a que emerjan distorsiones en el desarrollo de su actividad. Desde una perspectiva institucionalista, este artículo destaca que la existencia de información asimétrica al interior de las IES puede inducir al desarrollo de comportamientos oportunistas en los agentes involucrados en ausencia de incentivos o de adecuados sistemas de evaluación y control.

Un elemento que resulta vital para resguardar la calidad de las IES, es la existencia del desarrollo de mecanismos que fortalezcan el establecimiento de un sistema propicio de valores orientados a elevar y/o mantener adecuados estándares de calidad. Ello requiere generar por parte de las autoridades académicas los incentivos, motivaciones y controles necesarios para que los agentes se alineen en el proceso educativo. Junto con un adecuado marco institucional, debe haber una preocupación consciente por desarrollar y mantener una cultura y clima organizacional que fomente y fortalezca un proceso de enseñanza- aprendizaje.

En síntesis, la calidad de la educación en las IES es un asunto complejo. No existen elementos que de manera natural o sin esfuerzo garanticen que las IES a través del tiempo mejoren su calidad. Por el contrario, todo indica que existen ciertos factores que inducen a argumentar que, en caso de no tomar los resguardos adecuados, la calidad de las IES puede tender a deteriorarse. A este respecto, la existencia de políticas públicas en educación superior que contribuyan a reforzar la estructura institucional del sistema aparece como una creciente necesidad. 


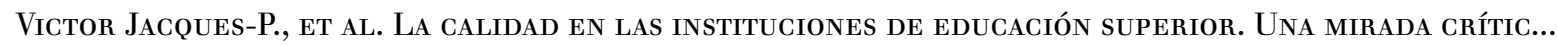

\section{ReFERENCIAS}

Armanet, P. (2004). Formación universitaria europea. Un ejemplo para Chile. Serie En Foco, 9. Recuperado de https:// bit.ly/2GxOJpp

Barrett, R. (2014). The values driven organization: Unleashing human potential for performance and profit. New York: Routledge.

Bouba-Olga, O., y Grossetti, M. (2008). Socio-économie de proximité. Revue d'Economie Régionale et Urbaine, 3, 311-328.

Caballero, G. (2011). Economía de las instituciones: de Coase y North a Williamson y Ostrom. Ekonomiaz, 77(2), $14-51$

Caballero, G., y Arias, X. (2013). De la Nueva Economía Institucional al análisis institucional moderno en las ciencias sociales. En X. Arias y G. Caballero (Eds.), Nuevo institucionalismo, gobernanza y politicas públicas. (pp. 17-40). Madrid: Centro de Investigaciones Sociológicas.

Coase, R. (1996). La naturaleza de la empresa (1937). En O. E. Williamson y S. G. Winter, La naturaleza de la empresa: origenes, evolución y desarrollo. (pp. 29-48). México: FCE.

Comisión Nacional de Acreditación (CNA). (2016). Gui\#a para la autoevaluacio\#n interna Universidades. Recuperado de https://www.cnachile.cl/

Fernández-Lamarra, N. (2012). Universidad y calidad en América Latina en perspectiva comparada. Interrogantes y desafíos. Revista da Avaliação da Educação Superior, 17(3), 661-688.

Fernández-Lamarra, N., y Coppola, N. (2008). La evaluación de la docencia universitaria en Argentina. Situación, problemas y perspectivas. Revista Iberoamericana de Evaluación Educativa, 1(3), 96-123.

Jacques, V., y Saona, P. (2011). El proceso de enseñanza-aprendizaje universitario: reflexiones a través de la teoría de agencia. Teoría de la educación: Educación y Cultura en la sociedad de la información, 12(1), 340-358.

Jensen, M. y Meckling, W. (1976). Theory of the firm: managerial behaviour, agency cost and ownership structure. Journal of Financial Economics, 3(4), 305-360.

Kahneman, D., y Tversky, A. (1973). On the psychology of prediction. Psychological review, 80(4), 237-251.

Lemaitre, M. (2015). Aseguramiento de la calidad: Una política y sus circunstancias. En A. Bernasconi (Ed.), La educación superior de Chile: Transformación, desarrollo y crisis. (pp. 295-344). Santiago, Chile: Ediciones UC.

López, A. (2016). La decisión de estudiar. Un análisis microeconómico desde el punto de vista del estudiante universitario. (Tesis de grado sin publicar). Universidad Austral de Chile, Chile.

Merton, R. (1995). Teoría y estructura sociales. México: FCE.

Mora, T., y Moreno, R. (2010). Specialization changes in European regions: the role played by externalities across regions. Journal of Geographical Systems, 12, 311-334.

North, D. C. (2014). Instituciones, cambio institucional y desempeño económico. México: FCE.

Quant, D. M., y Sánchez, A. (2012). Procrastinación, procrastinación académica: concepto e implicaciones. Revista Vanguardia Psicológica Clínica Teórica y Práctica, 3(1), 45-59.

Rodríguez, A., y Clariana, M. (2017). Procastinación en estudiantes universitarios: su relación con la edad y el curso académico. Revista Colombiana de Psicología, 26, 45-60.

Rodríguez, C., Martínez, G., Ponce, R., Contreras, E., Colina, R., y Cerritos, A. (2000). Una nueva estrategia para evaluar la calidad del desempeño docente en las IES. Revista de la Educación Superior ANUIES, 3,115.

Simon, H. (1962). El comportamiento administrativo. Estudio de los procesos decisorios en la organización administrativa. Madrid: Aguilar.

Simon, H. (2003). Racionalidad limitada en ciencias sociales: Hoy y mañana. En W. J. González (Ed), Racionalidad, historicidad y predicción en Herbert A. Simon (pp. 97-110). La Coruña: Netbiblo.

Williamson, O. (1989). Las instituciones Económicas del Capitalismo. México: FCE. 


\section{Notas}

[1]

North (1986) indica que las instituciones, junto con modificar "el conjunto de opciones a las que pueden acceder los seres humanos [también] cambian y por lo tanto alteran este conjunto disponible a lo largo del tiempo" (p. 1). Asimismo, el autor establece que las instituciones son una construcción social y en un momento dado pueden contribuir de mejor o peor manera al desempeño de los procesos que regulan.

Esto es independiente si el proceso de enseñanza aprendizaje se lleva a cabo mediante una educación basada en competencia o a través de una forma tradicional.

De acuerdo a Coase (1996), la empresa surge como una forma de evitar los costos de transacción que se generan en el mercado. La principal razón por la que es provechoso establecer una empresa y no recurrir al mercado "es, aparentemente, la existencia de un coste necesario por utilizar el mecanismo de precios [...] es el de descubrir cuáles son los precios relevantes. Este coste puede ser reducido, pero no eliminado, por la aparición de especialistas que vendan esta información. Además, están los costes por negociar y cerrar un contrato individual para cada transacción de intercambio" (p. 4).

[4]

Siguiendo a Simon (2003), los individuos en el mercado no toman decisiones óptimas pues se enfrentan a este con racionalidad limitada. "Las elecciones (choices) realizadas por la gente están determinadas no sólo por un objetivo general (overall goal) que sea consistente y por las propiedades del mundo externo, sino también por el conocimiento del mundo que tienen o dejan de tener quienes toman decisiones, de su habilidad o falta de habilidad para recordar ese conocimiento en el momento en que sea relevante, de saber sacar las consecuencias de sus acciones, de tener presentes las distintas posibilidades de actuación, de la capacidad para afrontar la incertidumbre" (p. 97).

Conforme a las tesis de Simon, la Nueva Economía Institucional "defiende que los individuos actúan con información incompleta y modelos subjetivamente deducidos, y asumen el modelo de racionalidad limitada, concibiendo al individuo como intencionalmente racional pero sólo de forma limitada" (Caballero y Arias, 2013, p. 31)

[6]

Para Coase, "lo que verdaderamente distingue a la empresa es que reemplaza el sistema de precios porque este supone costes, por lo que puede que las organizaciones jerárquicas afronten ciertas transacciones con menores costes" (Caballero, 2011, p. 19).

[7]

Coase (1996) establece que la empresa se justifica en la medida que sus costos de administración y coordinación de los recursos manera jerárquica son menores que los costos que implica el sistema de precios y la empresa deja de crecer cuando "una empresa tenderá a expandirse hasta que los costes por organizar una transacción suplementaria dentro de ella se hagan iguales a los costes por llevarla a cabo a través de un intercambio en el mercado libre" (p. 8).

[8]

En el conjunto de relaciones funcionales que se plantean en este trabajo, interesa destacar si la relación entre las variables es directa o inversa. En ese sentido, las variables pueden representar más o menos unidades de cantidad y/o calidad.

[9]

La OCDE, dentro de las acciones para fortalecer de las IES, señala que "los mecanismos institucionales para asegurar la calidad de los programas ofrecidos deben ser uno de los principales focos de la evaluación institucional” (Lemaitre, 2015, p. 324).

[10]

Al respecto resulta conveniente mencionar que, como establece Lemaitre (2015), que: "en otros países considerados en este estudio se insistió en que la institucionalización de procesos de diseño, planificación y control, tanto de la gestión como de la evaluación con fines de acreditación, así como la concentración de la información para la toma de decisiones habían conducido a la instalación de un estilo de gestión gerencial y burocrático, que muchas veces ignoraba criterios y prioridades académicas" (p. 335)

[11]

Entendiendo por valores aquellos elementos que son importantes para un grupo de personas, como por ejemplo la excelencia, la confianza, la honestidad o la rigurosidad (Barrett, 2014). 
[12]

Como indica Rodríguez et ál. (2000): “los docentes tienen en sus manos la misión más relevante de la educación superior. Por ello, reconocer el trabajo de aquellos capaces de crear un ambiente académico propio del aprendizaje, de estimular la imaginación, interés, capacidad de reflexión y deseos de superación de sus alumnos, e inculcar en ellos los principios y valores fundamentales de la educación universitaria es la forma más directa de aceptar que la enseñanza es la misión más relevante de las instituciones de educación superior" (p.1)

[13]

Este análisis sigue de manera análoga al modelo de competencia perfecta en economía. En este, a partir de ciertos supuestos que se pueden considerar ideales, se derivan ciertas conclusiones óptimas, que, si bien no se observan en el mundo real, sirven para que los organismos reguladores orienten los mercados a obtener resultados cercanos a los obtenidos por la competencia perfecta.

[14]

Para el caso chileno y como establece Armanet (2004), "uno de los rasgos del sistema de educación superior chileno es la tendencia a dilatar el tiempo necesario para la titulación, en parte porque los estudios toman más tiempo que el programado y también por los requisitos adicionales para recibir el título" (p. 5).

Si bien los enfoques de proximidad han sido aplicados en sectores diferentes al de la educación superior, su tratamiento acá resulta altamente pertinente por los fenómenos que intenta describir y explicar, totalmente atingentes a las situaciones de gobierno de las IES que tienen sistemas de elección de pares para cargos directivos.

[16]

La organización institucional de las IES para que sea efectiva y contribuya a la calidad debe incorporar en su gestión los procesos de planeación, evaluación y control del desempeño y de los resultados, sin embargo, en la medida que los cargos de dirección se van alternado entre los distintos docentes de una misma unidad académica, ello puede contribuir en ocasiones a bajar los estándares de los criterios de evaluación y control entre colegas. En el caso que esta actitud y comportamiento sea mayor en una organización de educación superior, mayor será el efecto colega.

Ciertamente existen instrumentos que se pueden aplicar, como la evaluación docente, las cuales permiten reducir en algún grado la falta de información por parte de los directivos.

A diferencia por ejemplo cuando se trata de medir el flujo de producción de un bien material realizado por un grupo de individuos en un lapso de tiempo determinado.

\section{[19]}

Este hecho a nivel micro es similar al problema de agencia. Al respecto, Jensen y Meckling (1976) se refieren a la relación de agencia al interior de una empresa cuando dos agentes, uno denominado como el principal (los propietarios del capital) encargan a otra persona al agente (gerente) la realización de algún servicio en su interés, concediéndole la autoridad necesaria al agente. El problema de agencia surge debido a que el agente al disponer de autoridad lleva cabo sus acciones en un entorno bajo el cual existe asimetría de información, lo que le otorga la posibilidad de perseguir además sus propios objetivos desalineándose de los intereses del principal.

[20]

De acuerdo a la teoría económica neoclásica, los agentes tienden a tener un comportamiento plenamente racional y optimizador, es decir, optan por la mejor opción que los conduce a alcanzar su objetivo. Sin embargo, desde el enfoque desarrollado por Simon (1962) sobre la racionalidad limitada, los individuos tienden a tomar decisiones por satisfacción más que por optimización, vale decir, privilegian la primera opción encontrada que les satisfaga en vez de encontrar la mejor entre un abanico de alternativas posibles.

Por cierto, este fenómeno puede darse también en el sentido inverso.

La procrastinación académica es un comportamiento prevalente en la población universitaria. Se estima que entre el $80 \%$ y el $95 \%$ de los estudiantes universitarios adopta conductas dilatorias en algún momento, el 75\% se considera a sí mismo procrastinador y el 50\% aplaza frecuentemente la dedicación a los estudios (Steel, citado por Rodríguez y Clariana, 2017, p. 47). 
Revista EduCaCión, 2019, 43(1), ISSN: 0379-7082 / 2215-2644

La procrastinación, sin duda es un fenómeno que puede estar presente en cualquier estamento en una IES, no es solo atingente a los estudiantes.

[24]

Debido a que estas variables influyen negativamente en la calidad (signo negativo de la relación), en la medida que aumentan cada una de estas variables, la gráfica CV se desplaza hacia abajo, lo cual significa que corresponde un nivel de calidad menor de las IES.

\section{BY-NC-ND}

